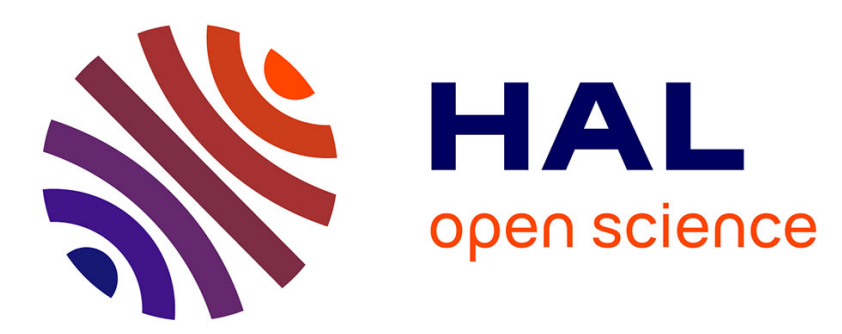

\title{
Uncertainty propagation combining robust condensation and generalized polynomial chaos expansion
}

Khaoula Chikhaoui, Najib Kacem, Noureddine Bouhaddi, Mohamed Guedri

\section{To cite this version:}

Khaoula Chikhaoui, Najib Kacem, Noureddine Bouhaddi, Mohamed Guedri. Uncertainty propagation combining robust condensation and generalized polynomial chaos expansion. International Modal Analysis Conference, Feb 2015, Orlando, FL, United States. hal-02991612

\section{HAL Id: hal-02991612 \\ https://hal.science/hal-02991612}

Submitted on 6 Nov 2020

HAL is a multi-disciplinary open access archive for the deposit and dissemination of scientific research documents, whether they are published or not. The documents may come from teaching and research institutions in France or abroad, or from public or private research centers.
L'archive ouverte pluridisciplinaire HAL, est destinée au dépôt et à la diffusion de documents scientifiques de niveau recherche, publiés ou non, émanant des établissements d'enseignement et de recherche français ou étrangers, des laboratoires publics ou privés. 


\title{
Chapter 24 \\ Uncertainty Propagation Combining Robust Condensation and Generalized Polynomial Chaos Expansion
}

\author{
K. Chikhaoui, N. Kacem, N. Bouhaddi, and M. Guedri
}

\begin{abstract}
Among probabilistic uncertainty propagation methods, the generalized Polynomial Chaos Expansion (gPCE) has 5 recently shown a growing emphasis. The numerical cost of the non-intrusive regression method used to compute the gPCE 6 coefficient depends on the successive Latin Hypercube Sampling (LHS) evaluations, especially for large size FE models, 7 large number of uncertain parameters, presence of nonlinearities and when using iterative techniques to compute the dynamic 8 responses. To overcome this issue, the regression technique is coupled with a robust condensation method adapted to the 9 Craig-Bampton component mode synthesis approach leading to computational cost reduction without significant loss of 10 accuracy. The performance of the proposed method and its comparison to the LHS simulation are illustrated by computing 11 the time response of a structure composed of several coupled-beams containing localized nonlinearities and stochastic design 12 parameters.

Keywords Robustness • Uncertainty • Generalized polynomial chaos expansion • Component mode synthesis $\bullet$ Meta- 14 model

\subsection{Introduction}

In structural mechanics and in practically all branches of industry, when parametric uncertainty is incorporated in the 17 mathematical model, its propagation is needed to evaluate how the randomness of input parameters affects the computed 18 outputs and to handle, consequently, more and more realistic behaviors. In a probabilistic framework, statistical approaches 19 such as the well-known Latin Hypercube Sampling (LHS) [1] use a great number of samples of random variables for 20 reasonable accuracy and therefore require a prohibitive computation time. On the other side, among other non statistical 21 approaches, the generalized polynomial chaos expansion (gPCE) [2] has shown a growing emphasis in recent years due to 22 its simple implementation and high performance. It combines polynomial basis vectors and deterministic coefficients, which 23 can be computed by means of intrusive or non-intrusive approaches [3]. In the context of the latter, more advantageous 24 than the former since it considers the original FE model as a black box, among other existing methods [4], regression 25 technique permits to estimate the gPCE coefficients by minimizing the difference between the referential LHS response and 26 the approximation, corresponding to a set of random variables chosen among combinations of Hermite polynomial roots. 27

Nevertheless, the most expensive part of its implementation lies in the successive LHS evaluations. To overcome this 28 issue, we focus, in this paper, on coupling the non-intrusive regression technique with a robust condensation method 29 adapted to the Craig-Bampton component mode synthesis (CBCMS). In fact, the prohibitive cost of prediction is due to: 30 the computational time of the full model using direct analysis, the computation of reduction basis for each random sample 31 while propagating uncertainties and the number of iterations necessary to obtain accurate approximations using iterative 32 techniques in presence of nonlinearities. Consequently, model condensation must be applied. Furthermore, the use of the 33 CBCMS [5, 6] allows considering some substructures, which are uncertain and/or containing localized nonlinearities, being 34 condensed independently of the others. Nevertheless, the main issue lies on forming a robust enriched condensed Craig- 35 Bampton Transformation (CBT) [6,7] which takes into account uncertainties and localized nonlinearities.

K. Chikhaoui $\bullet$ N. Kacem $(\bowtie) \cdot$ N. Bouhaddi

FEMTO-ST Institute, UMR 6174, Applied Mechanics Laboratory, University of Franche-Comté, 24 Chemin de l'Epitaphe, 25000 Besançon, France

e-mail: najib.kacem@femto-st.fr

K. Chikhaoui • M. Guedri

National High School of Engineers of Tunis (ENSIT), University of Tunis, 5 Avenue Taha Hussein, BP 56, Bâb Manara, Tunis, Tunisia 
In the literature, some works focus on coupling uncertainty propagation methods and condensation techniques in order to 37 attain a robust design. In [8], the authors implemented the CMS taking into account stochastic aspect to compute frequency 38 linear structures responses using stochastic spectral FE method (SSFEM). In [9], a reduced-order model (ROM) is integrated 39 into SSFEM, using a basis spanned by displacements and derivatives of displacements, and implemented to optimize the 40 shape of a linear shell structure. Afonso et al. [10] implemented Monte Carlo (MC) method and Probabilistic Collocation 41 Method (PCM) based on reduced-order modeling (ROM) approach incorporated via proper orthogonal decomposition 42 method (POD).

The main originality of this paper lies on combining gPCE and robust CBCMS in order to obtain a metamodel, which 44 allows computing stationary temporal solution of large size stochastic periodic structures containing local nonlinearities, 45 with high accuracy and low computational cost.

\subsection{Theoretical Backgrounds}

\subsubsection{Generalized Polynomial Chaos Expansion (gPCE)}

Thanks to the generalization of the PC approach [2], several types of random variables and orthogonal polynomials are taken 49 into account to develop its expansion. In this section, a compact form of the gPCE formulation is presented [2-4]. The gPCE 50 of second order random variable is a decomposition, truncated by retaining only terms of the polynomials with degree up to 51 $p$, of the form

$$
Y=\sum_{\alpha=0}^{P} y_{\alpha} \Phi_{\alpha}(\xi)=y^{T} \boldsymbol{\Phi}(\xi)
$$

where $y_{\alpha}$ are the unknown deterministic coefficients, $\Phi_{\alpha}$ the multivariate polynomials of $d$ independent random variables 53 $\xi=\left\{\xi_{i}(\theta)\right\}_{i=1}^{d}$ and $\alpha$ the multidimensional index such as $|\alpha|=\sum_{i=1}^{d} \alpha_{i}$.

The number of terms retained in Eq. 24.1 is defined as

$$
P+1=(d+p) ! / d ! p !
$$

Solving the gPCE consists on computing the coefficients $\mathrm{y}_{\alpha}$. Hence, the non-intrusive regression method is implemented, in 56 its standard form, minimizing the difference between the gPCE approximate solution and the exact one as follow

$$
\widetilde{y}=\underset{y}{\operatorname{Arg} \min } \frac{1}{N} \sum_{n=1}^{N}\left[\left\{Y^{(n)}\right\}-y^{T} \Phi\left(\xi^{(n)}\right)\right]^{2} \text {. }
$$

The solution considered as exact is a set of LHS responses $\left\{Y^{(n)}=y\left(\xi^{(n)}\right), n=1, \ldots, N\right\}$ computed corresponding to a 58 set of random variables $\Xi=\left\{\xi^{(n)}\right\}_{n=1}^{N}$ called experimental design (ED).

The final obtained approximate solution of Eq. 24.3 is of the form

$$
\widetilde{y}=\left(\boldsymbol{\Phi}^{T} \boldsymbol{\Phi}\right)^{-1} \boldsymbol{\Phi}^{T}\{y\}=\boldsymbol{\Phi}^{+}\{y\}
$$

where $\boldsymbol{\Phi}_{n j} \equiv\left(\Phi_{j}\left(\xi^{(n)}\right)\right)_{n=1, \ldots, N}$ is called the data matrix.

$$
j=0, \ldots, P
$$

A necessary condition for the numerical stability of the regression approximation is choosing an ED of size $N \geq P+162$ which ensures the well-conditioning of the matrix $\left(\boldsymbol{\Phi}^{T} \boldsymbol{\Phi}\right)$ which will be inverted. The selection of the ED is carried out using 63 two different methods; according to the first one, the ED is chosen randomly with respect to the probability distribution of the 64 random variables, the second method consists on selecting the ED, in a deterministic way, among Hermite polynomial roots 65 [3]. In fact, the roots of the Hermite polynomial of degree $p+1$ are at first computed, then all their possible combinations 66 $(p+1)^{d}$ are calculated and finally these roots combinations are classified such that the following variable 


\section{Author's Proof}

24 Uncertainty Propagation Combining Robust Condensation and Generalized Polynomial Chaos Expansion

$$
\zeta_{N}\left(\xi^{(n)}\right)=2 \pi^{-d / 2} \exp \left(-\frac{1}{2} \xi^{(n)^{2}}\right)
$$

is maximized or $\xi^{(n) 2}$ minimized. The roots combinations retained to create the ED are subject to another selection [4]. 68 Indeed, to ensure that the invertible matrix $\left(\boldsymbol{\Phi}^{T} \boldsymbol{\Phi}\right)$ is well-conditioned, a condition number $\kappa$ defined as

$$
\kappa=\left(\boldsymbol{\Phi}^{T} \boldsymbol{\Phi}\right)^{-1} \cdot \boldsymbol{\Phi}^{T} \boldsymbol{\Phi}
$$

must be minimized, where . is the 1-norm of the matrix. To the smallest value of $\kappa$ corresponds a number $N$ of roots 70 combinations which verify Eq. 24.5 and thus create the ED needed for gPCE coefficients.

Once obtained, the estimated coefficients give the final gPCE as a metamodel of the form

$$
\widetilde{Y}=\widetilde{\mathcal{M}}(X(\xi))=\sum_{\alpha=0}^{P} \widetilde{y}_{\alpha} \Phi_{\alpha}(\xi),
$$

In uncertainty analysis, some statistical quantities have to be calculated. The mean and the variance are respectively given 73 by $\widetilde{\mu}_{Y}=\widetilde{y}_{0}$ and $\widetilde{\sigma}_{Y}^{2}=\sum_{\alpha=0}^{P} \widetilde{y}_{\alpha}^{2}$.

Note that the $N$ successive deterministic FE evaluations needed for LHS method is the most expensive part of the gPCE 75 implementation especially for large size FE models, large number of uncertain parameters, presence of nonlinearities and 76 when using iterative techniques to compute dynamic structure responses. To overcome this issue, the regression technique is 77 coupled with a robust condensation method adapted to the CBCMS.

\subsubsection{Model Condensation}

In nonlinear dynamics, a mechanical system can generally be represented in the time domain by the following differential 80 equation

$$
\left\{\begin{array}{c}
{[M]\{\ddot{y}\}+[B]\{\dot{y}\}+\left\{f_{\text {int }}\right\}=\left\{f_{\text {ext }}\right\}} \\
\{y\}\left(t_{0}\right)=\{y\}_{0},\{\dot{y}\}\left(t_{0}\right)=\{\dot{y}\}_{0}: \text { initial conditions }
\end{array}\right.
$$

where $[M]$ and $[B]$ stand for the mass and damping matrices of the system, $\left\{f_{\text {ext }}\right\}$ the exciting force and $\left\{f_{\text {int }}\right\}=82$ $\left([K]+\left\{f_{N L}\right\}(\{y\},\{\dot{y}\})\right)\{y\}$ the internal force vector, $[K]$ is the stiffness matrix.

83

Modeling complex structures requires large size FE models for satisfying accuracy. To overcome the high computational 84 cost of the analysis, a reduced order model has to be designed using only few normal modes with respect to the frequency 85 range of interest. Therefore, the projection of the time response on an adequate condensation basis, considering the variable 86 transformation $\{y(t)\}=[T]\{q(t)\}$ to generalized coordinates, is needed. Hence, the equation of motion Eq. 24.8 becomes 87

$$
[T]^{T}[M][T]\{\ddot{q}(t)\}+[T]^{T}[B][T]\{\dot{q}(t)\}+[T]^{T}\left\{f_{\text {int }}\right\}_{r}=[T]^{T}\left\{f_{\text {ext }}\right\},
$$

where the index $r$ denotes the term reduced, $[T]^{T}[M][T]=[M]_{r},[T]^{T}[B][T]=[B]_{r}$, in this case, the internal force vector 88 is expressed as

$$
\left\{f_{i n t}\right\}_{r}=[\mathrm{T}]^{T}\left([\mathrm{~K}]+\left\{f_{N L}\right\}([\mathrm{T}]\{q\},[\mathrm{T}]\{\dot{q}\})\right)[\mathrm{T}]\{q\} .
$$

The time solution of the Eq. 24.9 can be approximated by using the Newmark nonlinear time integration scheme, which 90 allows expressing the displacement and the velocity at the instant $t_{n+1}$ as a function of their expression at $t_{n}$.

The implementation of the Newmark method permits to write the equation of motion Eq. 24.9 in an incremental form

$$
\left(\left[K_{e f f}\right]_{r}\right)_{n} \Delta\{q\}_{n}=\Delta\left(\left\{f_{e f f}\right\}_{r}\right)_{n}
$$


where $\left(\left[K_{\text {eff }}\right]_{r}\right)_{n}$ is the instantaneous (effective) stiffness matrix function of the tangent stiffness matrix $\left(\left[K_{T}\right]_{r}\right)_{n}=93$ $\partial\left(\left\{f_{\text {int }}\right\}_{r}\right)_{n} / \partial[\mathrm{T}]\{q\}_{n}$ obtained by applying the Newton iterative scheme.

\subsubsection{Robust Craig-Bampton Component Mode Synthesis Method (CBCMS)}

Dividing the complete structure into several components (substructures) is interesting in the case of presence of uncertainties 96 and localized nonlinearities in large size complex structures. It permits to apply the adequate condensation technique to each 97 component independently of the others.

In deterministic linear case, the reduced model is obtained using standard CBCMS [5, 6] of the linear system in its blocked 99 interface configuration. In this case, the CBT is defined, for a substructure $k$, as

$$
\{y\}^{k}=\left\{\begin{array}{c}
y_{j} \\
y_{i}
\end{array}\right\}^{k}=\left[\begin{array}{cc}
I_{j j} & 0 \\
\psi_{i j} & \varphi_{i}
\end{array}\right]^{k}\left\{\begin{array}{c}
q_{j} \\
q_{c}
\end{array}\right\}^{k}=\left[T_{C B}\right]^{k}\{q\}^{k}
$$

where $\left\{y_{i}\right\}^{k}$ are the interior coordinates transformed to the blocked junctions modal coordinates $\left\{q_{c}\right\}^{k},\left\{y_{j}\right\}^{k}$ are the junction 101 coordinates denoted as constraint coordinates $\left\{q_{j}\right\}^{k},\left[\psi_{i j}\right]=-K_{i i}^{-1} K_{i j}$ is the static subbasis which contains the constraint 102 modes, $I_{j j}$ is the identity matrix, and $\left[\varphi_{i}\right]$ is the dynamic one containing the truncated normal modes basis at blocked interfaces 103 $\left(\left\{y_{j}\right\}=0\right)$ of the corresponding component. Nevertheless, using standard CBT requires computing more and even all normal 104 modes for accurate results, which leads to a prohibitive computation cost.

In stochastic case with localized nonlinearities, the standard CBT $\left[T_{C B}\right]$ cannot satisfy the required accuracy and 106 robustness of the model. Therefore, adding a complementary basis $[\Delta T]$ is necessary in order to form an enriched CBT 107 (ECBT) $\left[T_{E C B}\right]$. The obtained basis, for each sub-structure, is thus of the form

$$
\left[T_{E C B}\right]^{k}=\left[T_{C B}: \Delta T\right]^{k}
$$

The complementary basis is a set of static residuals calculated according to the type of enrichment.

For each component $k$, to enrich the basis by taking into account stochastic aspect, the residual vectors are static responses 110 with correspondence to a set of residual forces $\left[F_{S}\right]$ presenting the stochastic effects. These forces are generated depending 111 on the stochastic zones of the mass and stiffness matrices [8]. The first complementary subbasis $\left[\Delta T_{S}\right]^{k}$ is thereafter obtained 112 and added to the standard Craig-Bampton one.

To take into account localized nonlinearity effects, another type of complementary subbasis $\left[\Delta T_{N L}\right]^{k}$ has to be created 114 [7] as a set of static responses corresponding to unit residual force vectors $\left\{F_{i}\right\}^{k}$ with respect to each nonlinear degree of 115 freedom (dof) $i$ for each component $k$.

The CBT can also be enriched if the external loading effect is considered [6]. Thus, an additional subbasis $\left[\Delta T_{E}\right]^{k}$ must 117 be computed using a set of unit static loadings $\left\{F_{E}\right\}^{k}$ imposed on internal excited substructure dofs.

Consequently, the final enriched basis has the following form

$$
\left[T_{E C B}\right]^{k}=\left[T_{C B} \vdots \Delta T_{S} \vdots \Delta T_{N L} \vdots \Delta T_{E}\right]^{k}=\left[\begin{array}{cccccccc}
I_{j j} & 0 & \vdots & 0 & \vdots & 0 & \vdots & 0 \\
\psi_{i j} & \varphi_{i} & \Delta T_{S} & & \Delta T_{N L} & \Delta T_{E}
\end{array}\right]^{k}
$$

Note that a singular value decomposition (SVD) is needed to ensure linear independence of the vectors forming each 120 complementary subbasis and also carried out on $\left[T_{E C B}\right]^{k}$ to ensure the linear independence of the subbases and thus the 121 well-conditioning of the ECBT. The synthesis of the complete structure requires finally the assembly of the different CBT 122 matrices according to the hypothesis of continuity of displacements at junction dofs.

\subsubsection{Robust Metamodel Combining CBCMS and gPCE}

The CBCMS and the gPCE methods were previously independently presented. The main aim of this paper is to couple them 125 in order to replace large size dynamical structures with considerably condensed and sufficiently accurate metamodel. Indeed, 126 we propose to compute the succession of $N$ deterministic responses of LHS simulations $\left\{Y^{(n)}=y\left(\xi^{(n)}\right), n=1, \ldots, N\right\}, 127$ 


\section{Author's Proof}

24 Uncertainty Propagation Combining Robust Condensation and Generalized Polynomial Chaos Expansion

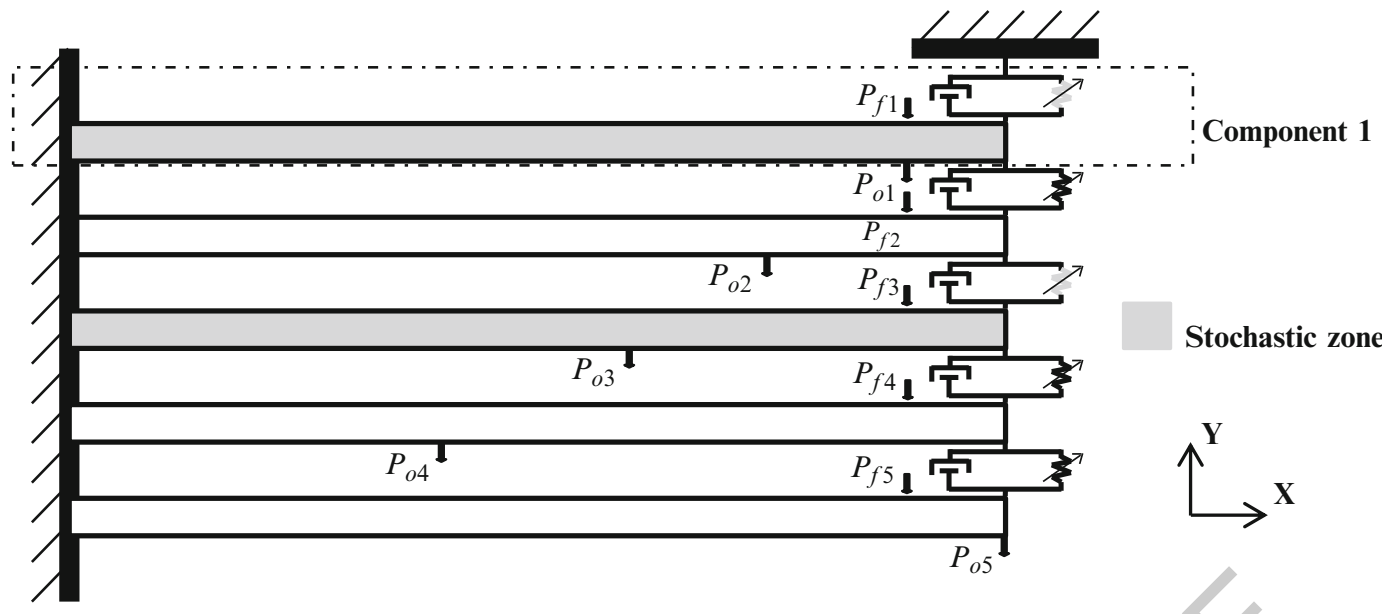

Fig. 24.1 Coupled beams structure

needed for the gPCE regression method implementation (Eq. 24.4), using the ECBT Eq. 24.14. The notion of robustness 128 consists, in our study, on satisfying two different criterions; the accuracy of the responses and the gain in terms of 129 computational time. To verify the first criterion, a set of temporal statistic moments $\mathscr{M}_{i}$, also called times indicators or 130 energy criterions, has to be calculated in order to quantify the response accuracy in terms of amplitude and periodicity errors. 131 These moments are expressed as [11]

$$
\mathcal{M}_{i}=\int_{-\infty}^{+\infty}\left(t-t_{s}\right)^{i} y(t)^{2} d t
$$

where $y(t)$ is the temporal response, $i$ the order of the moment and $t_{s}$ the temporal shift chosen in our case as $t_{s}=0$.

The total energy of the response $E=\mathcal{M}_{0}$ permits the verification of the accuracy in term of amplitude. $\mathcal{M}_{1} / \mathcal{M}_{0}=T$ 134 and $\mathcal{M}_{2} / \mathcal{M}_{0}-\left(\mathcal{M}_{1} / \mathcal{M}_{0}\right)^{2}=D^{2}$ are respectively the central time and the root mean square duration computed to verify 135 the accuracy of the response in term of periodicity.

A numerical application is presented in Sect. 24.3 in order to illustrate the main features of the proposed robust metamodel 137 designed to analyze the dynamic behavior in presence of uncertainties and localized nonlinearities.

\subsection{Numerical Application}

\subsubsection{Proposed Structure and Validation Process}

The proposed academic structure, Fig. (24.1), is composed of five identical beams loaded in pure flexion. The discretization 141 of each beam into 20 elements (two dofs per node: $v_{y}, \theta_{z}$ ) leads to a 200 dofs FE model. The beams are of rectangular 142 section with $b=3 \times 10^{-2} \mathrm{~m}$ and $h=1 \times 10^{-2} \mathrm{~m}$, length $L_{b}=5 \times 10^{-1} \mathrm{~m}$, Young modulus $E_{0}=2.1 \times 10^{11} P a, 143$ density $\rho_{0}=7800 \mathrm{~kg} \cdot \mathrm{m}^{-3}$ and Poisson's ratio $v=0.3$ and are submitted each one to a localized excitation force (N) 144 $P_{f}=10 \times \cos \left(2 \pi f_{1} t\right)$, where $f_{1}=82.96 \mathrm{rad} . \mathrm{s}^{-1}$ is the first eigenfrequency, according to the $v_{y}$ dof $(Y$ direction). The 145 five beams are coupled using five local dampers such as $c=10^{2} \mathrm{~N} . \mathrm{s} . \mathrm{m}^{-1}$, five linear springs $k_{0}=10^{6} \mathrm{~N} . \mathrm{m}^{-1}$ and nonlinear 146 ones $k_{N L}=10^{11} \mathrm{~N} . \mathrm{m}^{-1}$. Several observation points $P_{o j}(j=1,2, \ldots)$ are considered to evaluate the efficiency of the 147 proposed metamodel.

The stationary temporal response evaluation is done in the time interval $[0-0.5 \mathrm{~s}]$, divided into steps of $10^{-4} s$, in which 149 the stationary regime is already attained.

To apply the CBCMS method, the complete structure is divided into substructures. In fact, being in the case of periodic 151 structure, we propose to consider each set of coupling elements (localized damper, linear and nonlinear springs) and a beam 152 as a substructure (Fig. 24.1). The first and the third components are considered as two stochastic zones in which the Young 153 modulus of the beam and the linear coupling stiffness are supposed to be uncertain parameters such as 
Table 24.1 Model size and associated temporal moments

\begin{tabular}{|c|c|c|c|c|c|c|c|c|}
\hline \multirow{2}{*}{\multicolumn{2}{|c|}{ Methods }} & \multirow{3}{*}{$\begin{array}{c}\begin{array}{c}\text { Model } \\
\text { (dofs) }\end{array} \\
200\end{array}$} & \multicolumn{2}{|c|}{$\mathrm{E}$} & \multicolumn{2}{|c|}{$\mathrm{T}$} & \multicolumn{2}{|c|}{$\mathrm{D}^{2}$} \\
\hline & & & \multirow{2}{*}{$\begin{array}{c}\mathrm{E} \times 10^{4} \\
\left(\mathrm{~m}^{2} . \mathrm{s}\right)\end{array}$} & \multirow{2}{*}{$\begin{array}{c}\begin{array}{c}\text { Error } \\
(\%)\end{array} \\
0.00\end{array}$} & \multirow{2}{*}{$\begin{array}{c}\mathrm{T} \times 10^{2} \\
(\mathrm{~s}) \\
49.93\end{array}$} & \multirow{2}{*}{$\begin{array}{c}\begin{array}{c}\text { Error } \\
(\%)\end{array} \\
0.00\end{array}$} & \multirow{2}{*}{$\begin{array}{c}\mathrm{D}^{2} \times 10^{3} \\
(\mathrm{~s}) \\
82.03\end{array}$} & \multirow{2}{*}{$\begin{array}{c}\begin{array}{c}\text { Error } \\
(\%)\end{array} \\
0.00\end{array}$} \\
\hline LHS & LHS-REF & & & & & & & \\
\hline (A) & LHS-ECBT & 24 & 15.78 & 0.94 & 49.92 & 0.02 & 82.01 & 0.02 \\
\hline \multirow{4}{*}{$\begin{array}{c}\text { REG } \\
\text { (B) }\end{array}$} & REG-2-REF & 200 & 15.75 & 1.13 & 49.84 & 0.18 & 82.26 & 0.28 \\
\hline & REG-4-REF & 200 & 15.88 & 0.31 & 49.86 & 0.14 & 82.19 & 0.19 \\
\hline & REG-2-ECBT & 24 & 15.45 & 3.01 & 49.82 & 0.22 & 82.31 & 0.34 \\
\hline & REG-4-ECBT & 24 & 15.57 & 2.26 & 49.85 & 0.16 & 82.23 & 0.24 \\
\hline
\end{tabular}

$\mathrm{t} 3.2$

$$
E=E_{0}\left(1+\sigma_{E} \xi_{E}\right) \text { and } k=k_{0}\left(1+\sigma_{k} \xi_{k}\right)
$$

where $\xi_{E}$ and $\xi_{k}$ are two random variables of respectively uniform and lognormal probability distributions and $\sigma_{E}=\sigma_{k}=155$ $10 \%$ are the considered dispersions. The effect of the stochastic aspect of the uncertain parameters is shown using the MAC 156 (Modal Assurance Criterion) [6] matrix, which compares the normal modes of the deterministic model to the means of the 157 modes of the stochastic model computed with correspondence to each random variable.

The proposed metamodel results are discussed with respect to the responses considered as reference, computed using 159 LHS method using 1,000 samples of random variables. In fact, the process of evaluation of the metamodel efficiency is as 160 follow

(A) Implementing the 1,000 samples LHS method on: 1- the complete model (projection on complete eigenvectors basis) 162 denoted LHS-REF, 2- the reduced model (projection on the ECBT matrix) denoted LHS-ECBT;

(B) Implementing the regression gPCE: 1- on the complete model for two gPCE orders (2 and 4) denoted respectively 164 REG-2-REF and REG-4-REF, 2- combined with the ECBT (proposed metamodel) also for two gPCE orders, denoted 165 respectively REG-2-ECBT and REG-4-ECBT.

\subsection{Results and Discussion}

The ECBT matrix contains $n_{j}=8$ junctions dofs, $n_{i}=8$ retained normal modes and $n_{e}=n_{e S}+n_{e N L}+n_{e E}=2+5+1=8168$ enriching static residual vectors, where $n_{e S}$ corresponds to the stochastic enrichment, $n_{e N L}$ to the nonlinear one and $n_{e E}$ to the 169 external force enrichment. Hence, the size of the ECBT transformation matrix is $200 \times 24$, which signify that the reduction 170 ratio of the full problem is of $88 \%$ (200 dofs).

For a second order gPCE, the ED is of size 31 random variables combinations chosen with correspondence to the 172 conditions defined in Eqs. 24.5 and 24.6 among 81 Hermite polynomial roots combinations $\left((p+1)^{d}=3^{4}=81\right)$ and 173 then transformed with respect to the probability distributions (Table 24.1). For the fourth order, 84 random variables are 174 needed among 625 possible combinations.

Figure 24.2 shows the stochastic effect on the model normal modes through the MAC matrix. Figures 24.3 and 24.4176 illustrates the comparison between the means of stochastic velocities obtained by implementing the different methods with 177 correspondence to the above proposed process (A and B) at two chosen observation points $P_{o 1}$ and $P_{o 4}$.

The MAC matrix, presented in Fig. 24.2, illustrates the stochastic effect on the eight first modes retained for ECBT. 179 Indeed, the level of uncertainty is not high but sufficient to show the interest of the stochastic enrichment. Consequently, the 180 level of nonlinearities is chosen to be low in order to ensure the contribution of each type of enrichment in the ECBT. In fact, 181 high level of nonlinearities leads to a negligible stochastic enrichment contribution in the ECBT and thus enriching using 182 only nonlinear effect is sufficient.

The obtained results of different implemented models are compared in terms of accuracy and time consuming by means 184 of the CPU computation time and the temporal moments $E, T$ and $D^{2}$ (Tables 24.1 and 24.2). In fact, Table 24.1 recalls the 185 size of the problems to be solved and gives the values of the associated temporal moments and Table 24.2 contains different 186 computational time values. 


\section{Author's Proof}

24 Uncertainty Propagation Combining Robust Condensation and Generalized Polynomial Chaos Expansion

Fig. 24.2 MAC matrix

comparing the deterministic

normal modes and the means of

the stochastic ones

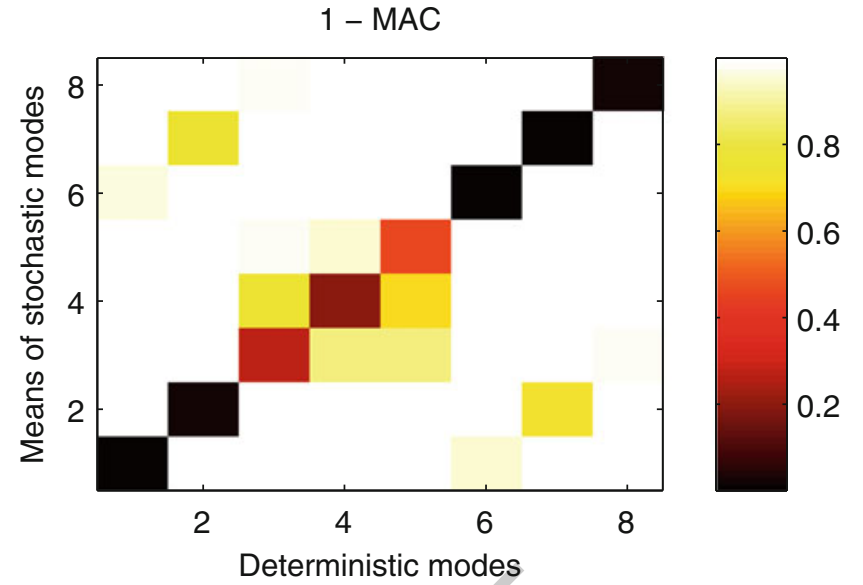

Po 1
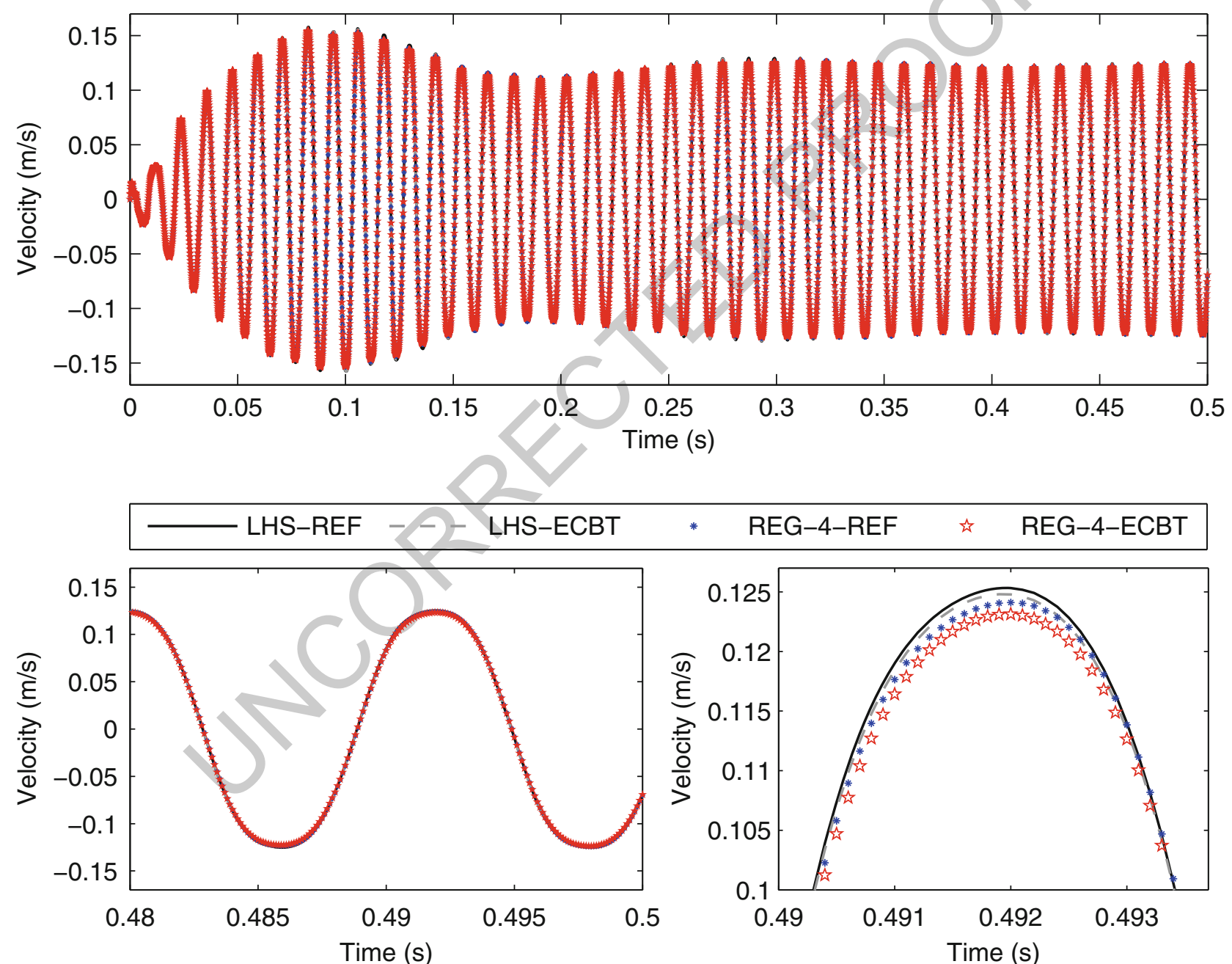

Fig. 24.3 Mean of the stochastic velocity at the observation point $P_{o 1}$ computed on the reference (complete) model (REF) and the condensed one (ECBT) using LHS and fourth order gPCE methods

The results displayed in Figs. 24.3 and 24.4 show that the proposed metamodel can replace the original one without a 188 significant loss of accuracy with respect to the reference results computed by LHS method on the full structure. Note that the 189 higher the order of the gPCE is, the more accurate the results are, the longer the required computational time is, as shown in 190 


\section{Author's Proof}

K. Chikhaoui et al.

Po 4
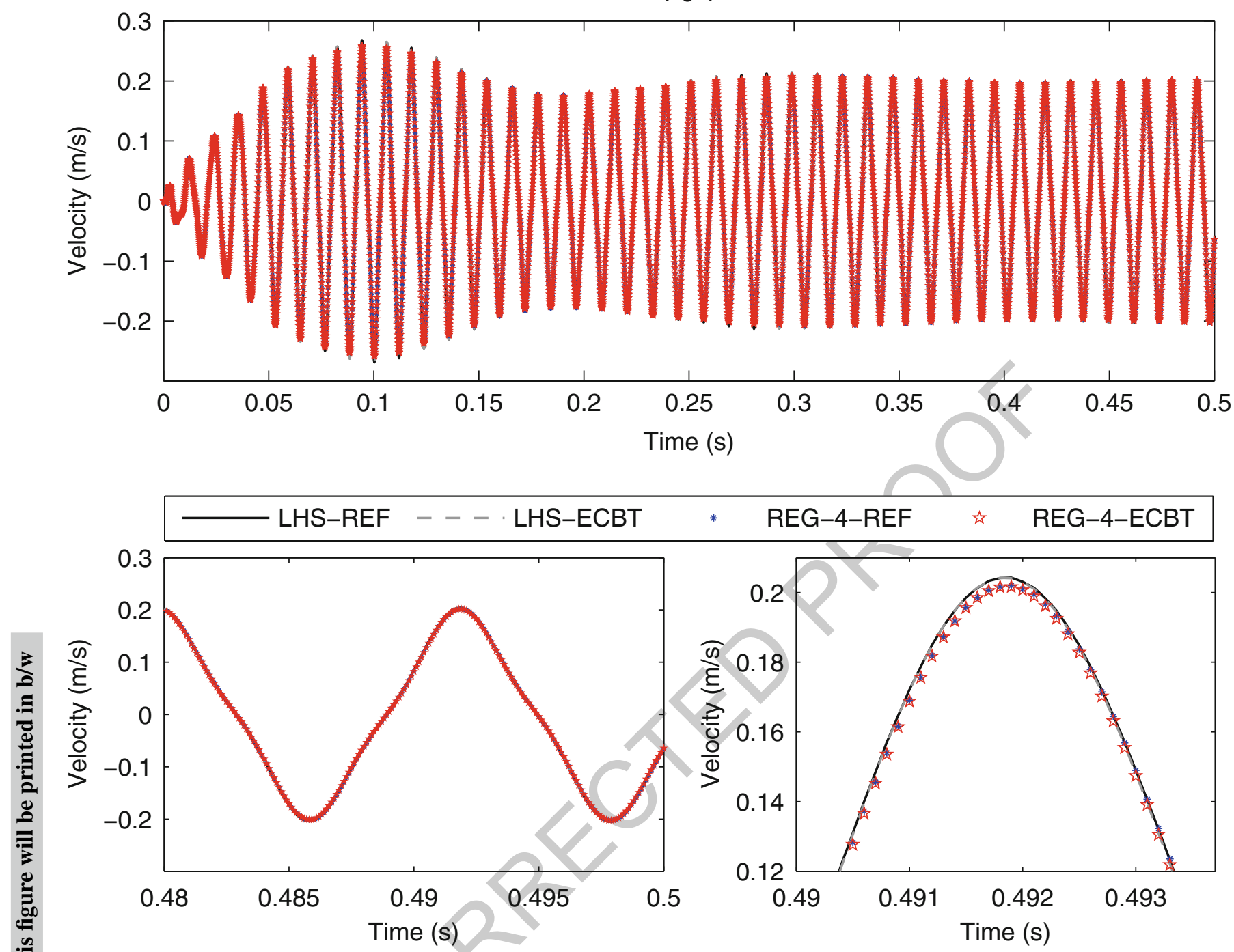

Fig. 24.4 Mean of the stochastic velocity at the observation point $P_{o 4}$ computed on the reference (complete) model (REF) and the condensed one (ECBT) using LHS and fourth order gPCE methods

Table 24.2 Computational CPU time

\begin{tabular}{|c|c|cc|}
\hline \multicolumn{2}{|c|}{ Methods } & CPU (min) & CPU (\%) \\
\hline LHS & LHS-REF & 6049.10 & 100 \\
\cline { 2 - 4 } (A) & LHS-ECBT & 4405.13 & 72.82 \\
\hline & REG-2-REF & 201.19 & 3.33 \\
REG & REG-4-REF & 463.99 & 7.67 \\
\cline { 2 - 4 } (B) & REG-2-ECBT & 155.40 & 2.57 \\
& REG-4-ECBT & 399.61 & 6.61 \\
\hline
\end{tabular}

t5.1

Table 24.2. Only the fourth order gPCE approximations are presented, the second order gPCE results are discussed through 191 their temporal moments presented in Table 24.1. The ECBT efficiency can be at first evaluated comparing the two first curves 192 (Figs. 24.3 and 24.4) and the two first lines of Table 24.1, which show that the amplitude and periodicity errors between the 193 results of the LHS method on full and condensed model are very small.

Table 24.2 shows that the computation time can be reduced by $93.4 \%$ when implementing the proposed metamodel, and 195 it increases when the gPCE order is higher. Note that despite the fact that the example is not sufficiently representative in size 196 and uncertain parameter number to show the efficiency of the CBCMS, the gain of nearly $30 \%$ attained when implementing 197 


\section{Author's Proof}

24 Uncertainty Propagation Combining Robust Condensation and Generalized Polynomial Chaos Expansion

ECBT with LHS or gPCE reflects more than $30 \mathrm{~h}$ of time reduction (1\% of gain is $61 \mathrm{~min}$ ). For larger size examples, and 198 also when using properly optimized algorithms, the robust CBCMS contribution should be more illustrated.

\subsection{Concluding Remarks}

In this paper, a robust metamodel is proposed in order to approximate structure behaviors in presence of uncertainties and 201 localized nonlinearities. This metamodel is obtained by the combination between the parametric uncertainty propagation 202 method and a robust CMS approach. The efficiency of the metamodel was successfully evaluated on the temporal response 203 approximations of the coupled beams structure with respect to the reference LHS method results, thanks to different verified 204 robustness criterions. The reduction in terms of time consuming and model size using the comparison of the computation 205 times and temporal moments prove the efficiency and the usefulness of the proposed metamodel for the robustness analysis 206 of large size nonlinear stochastic model.

\section{References}

1. Helton JC, Davis FJ (2003) Latin hypercube sampling and the propagation of uncertainty in analyses of complex systems. Reliab Eng Syst Saf 209 81:23-69

2. Xiu D, Karniadakis GE (2002) The Wiener-Askey polynomial chaos for stochastic differential equations. SIAM J Sci Comput 24(2):619-644 211

3. Berveiller M (2005) Eléments finis stochastiques : Approches intrusive et non intrusive pour des analyses de fiabilité, thèse de Doctorat. 212 Université BLAISE PASCAL - Clermont II, Aubière

4. Blatman G, Sudret B (2010) An adaptive algorithm to build up sparse polynomial chaos expansions for stochastic finite element analysis. 214 Probab Eng Mech 25:183-197

5. Craig RR Jr, Bampton MCC (1968) Coupling of substructures for dynamic analyses. AIAA J 6(7):1303-1319

6. Lombard JP (1999) Contribution à la réduction des modèles éléments finis par synthèse modale, thèse de Doctorat. Université de Franche- 217 Comté, France

7. Bouazizi ML, Guedri M, Bouhaddi N (2006) Robust component modal synthesis method adapted to the survey of the dynamic behavior of 219 structures with localized nonlinearities. Mech Syst Signal Process 20:131-157

8. Guedri M, Bouhaddi N, Majed R (2006) Reduction of the stochastic finite element models using a robust dynamic condensation method. 221 J Sound Vib 297:123-45

9. Maute K, Weickum G, Eldred M (2009) A reduced-order stochastic finite element approach for design optimization under uncertainty. Struct 223 Saf 31:450-459

10. Afonso SMB, Motta RS (2013) Structural optimization under uncertainties considering reduced-order modeling, 10th world congress on 225 structural and multidisciplinary optimization, Orlando, 19-24 May 2013

11. Hemez FM, Doebling SW (2003) From shock response spectrum to temporal moments and vice-versa, international modal analysis conference 227 (IMAC-XXI), Kissimmee, 3-6 Feb 2003 